Provided for non-commercial research and education use. Not for reproduction, distribution or commercial use.

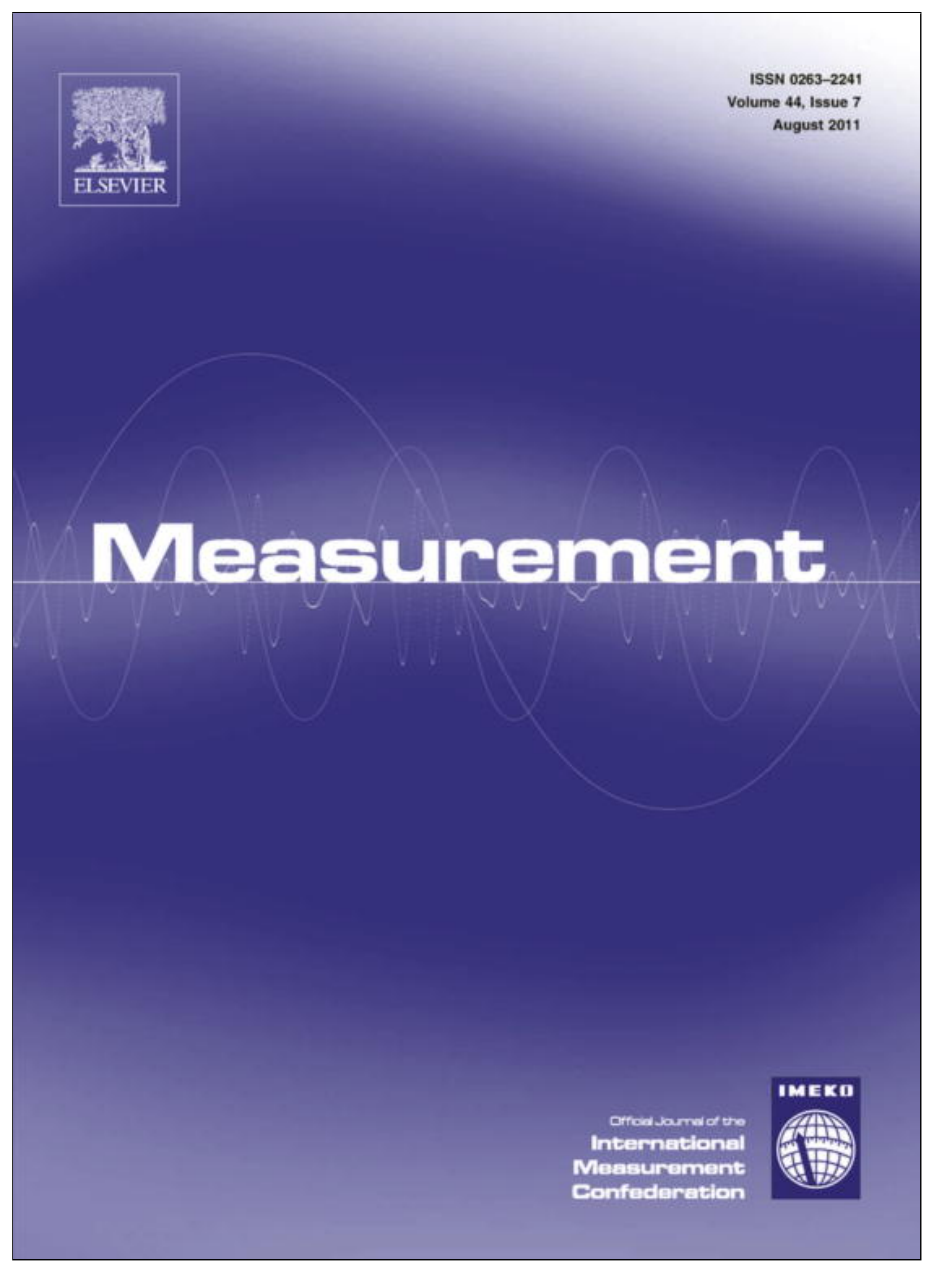

This article appeared in a journal published by Elsevier. The attached copy is furnished to the author for internal non-commercial research and education use, including for instruction at the authors institution and sharing with colleagues.

Other uses, including reproduction and distribution, or selling or licensing copies, or posting to personal, institutional or third party websites are prohibited.

In most cases authors are permitted to post their version of the article (e.g. in Word or Tex form) to their personal website or institutional repository. Authors requiring further information regarding Elsevier's archiving and manuscript policies are encouraged to visit:

http://www.elsevier.com/copyright 


\title{
Fast genetic algorithm for roundness evaluation by the minimum zone tolerance (MZT) method
}

\author{
Andrea Rossi, Michele Antonetti, Matteo Barloscio, Michele Lanzetta* \\ Department of Mechanical, Nuclear and Production Engineering, University of Pisa, Via Bonanno Pisano 25B, 56126 Pisa, Italy
}

\section{A R T I C L E I N F O}

\section{Article history:}

Received 25 May 2010

Received in revised form 28 February 2011

Accepted 31 March 2011

Available online 3 April 2011

\section{Keywords:}

Minimum zone tolerance (MZT)

Roundness error

Genetic algorithm

CMM

\begin{abstract}
A B S T R A C T
According to ISO 1101, “A geometrical tolerance applied to a feature defines the tolerance zone within which that feature shall be contained".

The main goal of the minimum zone tolerance (MZT) method is to achieve the best estimation of the roundness error, but it is computationally intensive. This paper describes the application of a genetic algorithm (GA) to minimize the computation time in the evaluation of CMM roundness errors of a large cloud of sampled points.

Computational experiments have shown that by selecting the optimal GA parameters, namely a combination of the five genetic parameters related to population size, crossover, mutation, stop condition, and search space, the computation time can be reduced by up to one order of magnitude, allowing real-time operation.

Optimization has been tested using seven CMM samples, obtained from different machining features. The performance of the optimized algorithm has been validated using four benchmark samples from the literature and with certified samples.
\end{abstract}

(c) 2011 Elsevier Ltd. All rights reserved.

\section{Introduction}

Roundness is one of the main functional product features in machining and assembly when handling, positioning and mating are involved.

The most used methods to determine form errors, particularly roundness errors, are:

- least-squares (LSQ) method,

- maximum inscribed circle (MIC) method,

- minimum circumscribed circle (MCC) method, and

- minimum zone tolerance (MZT) method.

With the LSQ method, a circle is fitted to the roundness profile using the least-squares method. The center of that circle is used to fit the smallest circumscribed and the largest inscribed circles to the roundness profile. The radial

\footnotetext{
* Corresponding author. Tel.: +39 050 2218122; fax: +39 0502218140

E-mail address: lanzetta@unipi.it (M. Lanzetta).
}

separation between these two circles is the roundness error. The LSQ method [1] is the most popular method for evaluating the roundness error. This method has been widely used in CMMs in view of the simplicity of its application. For the same reason, the associated derived center of the least-squares reference circle (LSC) is often used as the starting point in many search methods.

With the MIC and MCC methods [2], the maximum inscribed and the minimum circumscribed reference circles are respectively found.

The MIC (MCC) method also detects the smallest circumscribed (largest inscribed) circle that is concentric to the reference circle. Their radial distance represents the maximum inscribed (minimum circumscribed) roundness error.

According to [3], the Tschebyscheff approximation is the correct approach to determine standardized form tolerances like roundness, flatness or cylindricity deviations, leading to MIC/MCC method for roundness profiles.

Unfortunately the roundness error evaluation with the above methods is not the optimum, resulting in a possible 
overestimation. If the roundness error after evaluation according to the above methods is smaller than the roundness tolerance, the part is always within the tolerance and has to be accepted when testing conformance with specification. If it is not, although CMM algorithms successfully reject defective parts, they may also reject parts that satisfy the design tolerances. For this reason, alternative methods able to achieve a better roundness estimate may produce economical benefits.

MZT methods deem the minimum zone reference circles (MZCI [4]), i.e. the two concentric circles with minimum radial separation that contain the roundness profile. The radial separation between these two circles is the roundness error. By definition, MZT methods produce optimal as well as correct solutions, because the reference circles have the minimum radial separation. Hence, it detects fewer out of tolerance parts compared to the LSQ MIC and MCC methods. International Organization for Standardization [5] suggests that the MZT criterion should be applied to evaluate roundness.

The drawback of the techniques based on the MZT method is that it requires the solution of a non-linear problem. The complexity and consequently the computation time of MZT algorithms are very sensitive to the number of sample points. Due to the problem complexity increase with the dataset size, suitable optimization algorithms are required.

In previous works, the authors developed a fast algorithms for small samples to assess the kind of deviation on the roundness profile in order to detect critical points such as peaks and valleys $[6,7]$.

Among the fastest roundness error evaluation methods to implement, already available in the literature based on the MZT, are the steepest descent algorithm, which has been applied for roundness evaluation [8], and a twodimensional simplex search method to evaluate several form features, including roundness [9]. However, they do not guarantee a global optimal solution for non-convex problems.

Xiong [10] develops a general mathematical theory, a model and an algorithm for different kinds of profiles, including roundness where the linear programming method and exchange algorithm are used. As limaçon approximation is used to represent the circle, optimality of the solution is however, not guaranteed.

A strategy based on geometric representation for minimum zone evaluation of circles and cylinders is proposed by Lai and Chen [11]. The strategy employs a non-linear transformation to convert a circle into a line and then uses a straightness evaluation schema to obtain minimum zone deviations for the feature concerned. This is an approximation strategy to minimum zone circles.

Wang et al. [12] present a generalized non-linear optimization procedure based on the developed necessary and sufficient conditions to evaluate roundness error.

Wang [13] proposes a general-purpose algorithm for constrained non-linear optimization problems for minimum zone evaluation of form tolerances. He uses a technique similar to the sequential quadratic programming method, verifying that an optimal solution to the quadratic programming problem can be obtained in a finite number of iterations.

Samuel and Shunmugam [14] establish a minimum zone limaçon based on computational geometry to evaluate roundness error; with geometric methods, exact global optimal solutions are found by exhaustively checking every local minimum candidate.

Other researchers like Chang and Lin [15] use the Monte Carlo simulation method to evaluate roundness errors.

Another approach is based on the Voronoi diagram as described by Roy and Zhang [16]; the method yields a very accurate measurement of the roundness error, but it is computationally intensive.

On the opposite Xianqing et al. [17] propose a mesh based method with starting center on the LSC, where the convergence depends on the number of mesh cross points, representing a compromise between accuracy and speed.

As for genetic algorithms (GAs) to find the solution of the MZT problem, as proposed in this paper, Sharma et al. [18] use a standard GA for the evaluation of multiple form tolerance classes such as straightness, flatness, roundness, and cylindricity. There is no need to optimize the algorithm performance, choosing the parameters involved in the computation, because of the small dataset size (up to 100 sample points).

Wen et al. [19] implement a GA in real-code, with only crossover and reproduction operators applied to the population. Thus in this case mutation operators are not used. The algorithm proposed is robust and effective, but it has only been applied to small samples.

Among metaheuristic methods to approach the Chebyshev best fit criterion are swarm particle optimization (SPO) [20] and one sided best fit by simulated annealing (SA) [21] with encouraging results.

MZT, despite its complexity, is a potential replacement of traditional methods in industry for the mentioned economical benefits. However, for on-line inspection the computation time becomes a key factor. So not only the roundness error needs to be minimized, but also the computation time must be a performance index.

GAs differ considerably in conception from other search methods. The basic difference is that while other methods always process single points in the search space, GAs maintain a population of potential solutions, and so perform an multi-directional implicitly parallel search.

The proposed GA has been tested on larger samples than available in the literature (1800 sample points).

Larger samples available by CMM scanning techniques allow reducing the form error estimation, but they would require optimal GA parameters for faster convergence. On the other hand, larger samples allow an effective tuning in order to minimize the computation time, because the GA parameters optimized on larger samples may provide useful information about the behavior of GAs with few hundreds or less sample points, like in standard CMM applications.

The optimal GA parameters have been experimentally evaluated on seven samples and the performance validated on four samples from the literature and with certified samples. 


\section{The MZT method}

In the MZT method, the unknown are the $(x, y)$ coordinates of the associated derived center of the minimum zone reference circles of the roundness profile (MZCI [4]). $\mathrm{MZCI}$ is formed by two concentric circles enclosing the roundness profile, the inner minimum zone reference circle and the outer minimum zone reference circle, having the least radial separation. The difference between the inner minimum zone reference circle and the outer minimum zone reference circle is the minimum zone error $(M Z E)$. MZE is the target parameter of our optimization algorithm as a function of $(x, y)$.

Given an extracted circumferential line $r(x, y, \theta)$, with $\theta$ $\in(0,2 \pi]$, of a section perpendicular to the axis of a cylindrical feature, the roundness error $R(x, y)$ is defined by:

$R(x, y)=O C(x, y)-I C(x, y),(x, y) \in E_{r(x, y, \theta)}$

where $O C(x, y)$ and $I C(x, y)$ are the radii of the reference circles of center $(x, y)$, and $E_{r(x, y, \theta)}$ is the area enclosed by $r(x, y, \theta)$ :

$O C(x, y)=\max _{\theta \in(0,2 \pi]} r(x, y, \theta)$

$I C(x, y)=\min _{\theta \in(0,2 \pi]} r(x, y, \theta)$

As a CMM scans the roundness profile by sampling a finite number, $n$, of equally-spaced points $\theta_{i}$ of the extracted circumferential line $\left(\theta_{i}=i \times \frac{2 \pi}{n}, i=1, \ldots, n\right)$, the $O C(x, y)$ and the $I C(x, y)$ are evaluated by:

$$
\begin{aligned}
& \text { OC }(x, y)=\max _{\theta_{i}=i \times \frac{2 \pi}{n}, i=1, \ldots, n} r\left(x, y, \theta_{i}\right) \\
& I C(x, y)=\min _{\theta_{i}=i \times \frac{2 \pi}{n}, i=1, \ldots, n} r\left(x, y, \theta_{i}\right)
\end{aligned}
$$

Fig. 1 shows the mentioned features for a given $(x, y)$.

MZE is evaluated by applying the MZT data-fitting method to solve the following optimization problem:

$$
\begin{aligned}
\text { MZE } & =\min _{(x, y) \in E_{r(x, y, \theta)}} R(x, y) \\
& =\left\{\begin{array}{l}
\min \left[\max _{\theta_{i}=i \times \frac{2 \pi}{n}, i=1, \ldots, n} r\left(x, y, \theta_{i}\right)-\min _{\theta_{i}=i \times \frac{2 \pi}{n}, i=1, \ldots, n} r\left(x, y, \theta_{i}\right)\right] \\
\text { subject to }(x, y) \in E_{r\left(x, y, \theta_{i}\right)}
\end{array}\right.
\end{aligned}
$$

where $E_{r\left(x, y, \theta_{i}\right)}$ is a restricted area in the convex envelopment of the $n$ equally-spaced sample points, i.e. the search space.

\section{Genetic parameters}

GAs were proposed for the first time by Holland [22] and constitute a class of search methods especially suited for solving complex optimization problems [23].

GAs maintain a population of individuals that are represented by their chromosomes, which are made of genes; GAs operate on the genes, which represent the inheritable properties of the individuals, by means of genetic operators. In each generation, the genetic operators are applied to selected individuals from the current population in order to create a new generation according to the iterative standard scheme in Fig. 2 also used in this work.

A fitness function is assigned to an individual in order to reflect how well a solution fulfills the requirements of the given problem. The fitness function also makes it possible to evolve only good solutions.

The standard genetic operators are the following:

Selection: during this operation, a solution has a probability of being selected according to its fitness. One

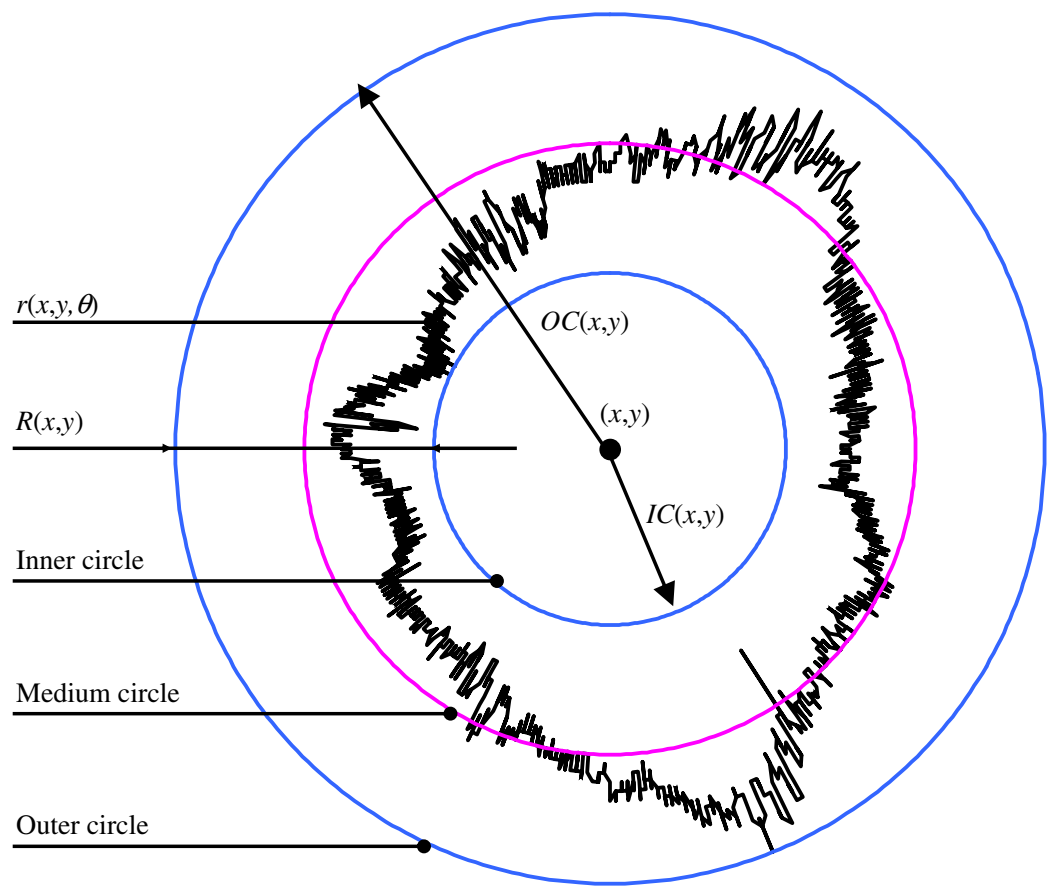

Fig. 1. Roundness profile of sample no. 1 (Table 2 ) with 1800 equally-spaced CMM sample points and reference circles: inner, medium, and outer circles for a given associated derived center $(x, y)$. 


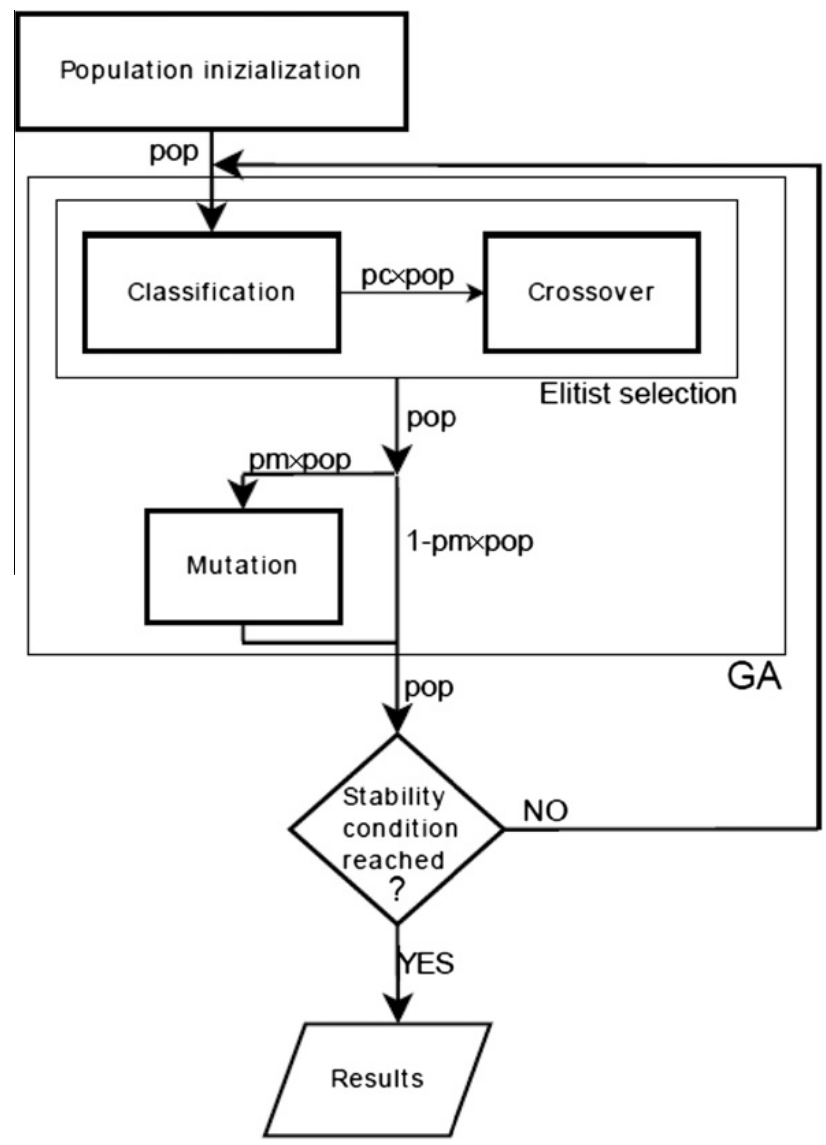

Fig. 2. Standard GA scheme.

mechanism that allows fitness proportional selection is the roulette wheel procedure. Each individual has a chance to be selected, which is directly proportional to its fitness in the roulette wheel. Tournament selection is another reproduction mechanism. Tournament selection involves running several tournaments among a few individuals randomly chosen from the population. The individual with the best fitness is selected for crossover. If the tournament size is larger, weak individuals have a smaller chance of being selected. A third reproduction mechanism is elitist selection. With this method, the individuals are ordered on the basis of their fitness function; the best individuals produce offspring. The next generation will be composed of the best chromosomes chosen between the set of offspring and the previous population.

Crossover: new individuals are generally created as offspring of two parents. This method allows inheriting genes from parents with high fitness by the selection mechanism. One or more so-called crossover points are selected within the chromosome of each parent, at the same place in each. The parts delimited by the crossover points are then interchanged between the parents. The resulting individuals are the offspring. These kinds of crossovers are controlled by the crossover probability. Beyond one point and multiple point crossover, there exist more sophisticated crossover types. The so-called knowledge-augmented crossover operator constructs offspring from parents by making use of domain knowledge related to the given problem. Another crossover mechanism is arithmetic crossover, which generates offspring as a component-wise linear combination of the parents.

Mutation: a new individual is created by making modifications to one selected individual. The modifications consist of changing one or more values in the representation, or in adding/deleting parts of the representation. In GAs, mutation is a source of variability, and is applied in addition to selection and crossover. This method prevents the search to be trapped only in local solutions. The mutation probability controls the operator behavior. Stop criterion: the algorithm has an iterative behavior and needs a stop condition to end the computation. Possible criteria include: overcoming a predefined threshold for the fitness function or iteration number or their combinations.

\subsection{GA for roundness error evaluation}

A GA to solve the optimization problem (6) for a twodimensional search space entails a population of chromosomes made of pairs of coordinates (their genes).

These genes represent respectively the $x$ and $y$ coordinates of the center of the reference circles of radii $O C(x, y)$ and $I C(x, y)$ (Fig. 1). A pair of genes (chromosome) is a candidate for the associated derived center. The crossover operator recombines genes (parents) in order to find better candidates of the associated derived center (offspring).

For three-dimensional features such as spheres and cylinders, a third coordinate $(z)$, and consequently a third gene, are required.

A fitness function defines the goodness of each chromosome $(x, y)$ as solution of (6). As shown by (1), the fitness function is represented by the roundness error $R(x, y)$.

By applying genetic operators, the data-fitting algorithm evolves from generations of 2D coordinates implicitly exploring the search space, until a stop condition establishes the convergence to the associated derived center.

\section{The proposed GA to assess the MZT to large samples}

GAs are able to provide a (local) optimum in all cases. However, to improve the convergence speed towards more accurate solutions, particularly with large samples, the genetic parameters need to be tuned.

In this work we describe the configuration of the genetic parameters in order to optimize the computation time for the evaluation of a target roundness error.

The GAs performance are influenced by the following five main parameters as detailed in Table 1:

- population size (pop),

- probability of crossover $(p c)$,

- probability of mutation ( $p m)$,

- stability condition $(N)$; and

- search space size $(E)$. 
The initial population is randomly selected around the LSC, one individual being the LSC itself, to restrict the search space. The LSC is used as the starting point also in other non GA methods like the steepest descent algorithm in [8] and the mesh based method in [17].

Table 1

Genetic operators, their parameters and mechanisms.

\begin{tabular}{|c|c|c|}
\hline $\begin{array}{l}\text { Genetic } \\
\text { operator }\end{array}$ & Parameter & Remark \\
\hline - & pop & Population size \\
\hline Selection & - & Elitist selection \\
\hline Crossover & $p c$ & $\begin{array}{l}\text { One point crossover of the } p c \times p o p \\
\text { parents' genes (i.e. coordinates) at } \\
\text { each generation }\end{array}$ \\
\hline Mutation & pm & $\begin{array}{l}p m \times p o p \text { individuals are modified by } \\
\text { changing one gene (i.e. coordinate) } \\
\text { with a random value }\end{array}$ \\
\hline Stop criterion & $N$ & $\begin{array}{l}\text { The algorithm computes } N \\
\text { generations after the last best } \\
\text { roundness error evaluated rounded } \\
\text { off to the fourth decimal digit } \\
(0.1 \mu \mathrm{m})\end{array}$ \\
\hline Search space & $E$ & $\begin{array}{l}\text { Initial population randomly selected } \\
\text { within }\end{array}$ \\
\hline
\end{tabular}

Table 2

Experimental set [24].

\begin{tabular}{llll}
\hline Dataset & Diameter $(\mathrm{mm})$ & Process & Material \\
\hline 1 & 10 & Drilling & Aluminum alloy \\
2 & 10 & Milling & Steel \\
3 & 14 & Turning & Steel \\
4 & 17 & Drilling & Marble \\
5 & 23 & Turning & Steel \\
6 & 25 & Turning & Aluminum alloy \\
7 & 39 & Turning & Steel \\
\hline
\end{tabular}

To stop the algorithm, the stability condition with the mechanism described in Table 1 is proposed because the computation time is the main focus of this work. The selection of $N$ is a compromise between two opposites targets: too small a value leads to an early stop of the evaluated generations with a non-optimal solution; too big a value guarantees the optimal solution, but causes an unacceptable increase in the computation time.

The total computation time can be considered as an index of the algorithm performance for a given workstation.

GA was implemented on Intel ${ }^{\circledR}$ Core $^{\mathrm{TM}} 2$ Duo CPU P8400 @ 2.26 GHz Workstation, using Visual C++ 5.0.

\subsection{Large samples}

Different materials (steel, aluminum, and marble), geometrical features and processes (drilling and internal turning) have been considered (Table 2).

For each dataset, the roundness profile was obtained by scanning a cloud of 1800 equally-spaced points on the circumference $\left(0.2^{\circ}\right)$. The samples no. $1-7$ are also shown in Figs. 1 and 3.

\subsection{Explorative phase}

The genetic parameters have been preliminarily explored over the range pointed out by the literature (Table 3 ) on the samples of Table 2:

- pop, four values: 200, 300, 400 and 500; the range 200 to 500 is higher than proposed in the literature because of the higher complexity coming from the larger samples;

- $p c, 14$ equally-spaced values between 0.01 and 0.9 ;

- pm, 14 equally-spaced values between 0.001 and 0.04 ;

- $N$ was conservatively set as high as 150 ;
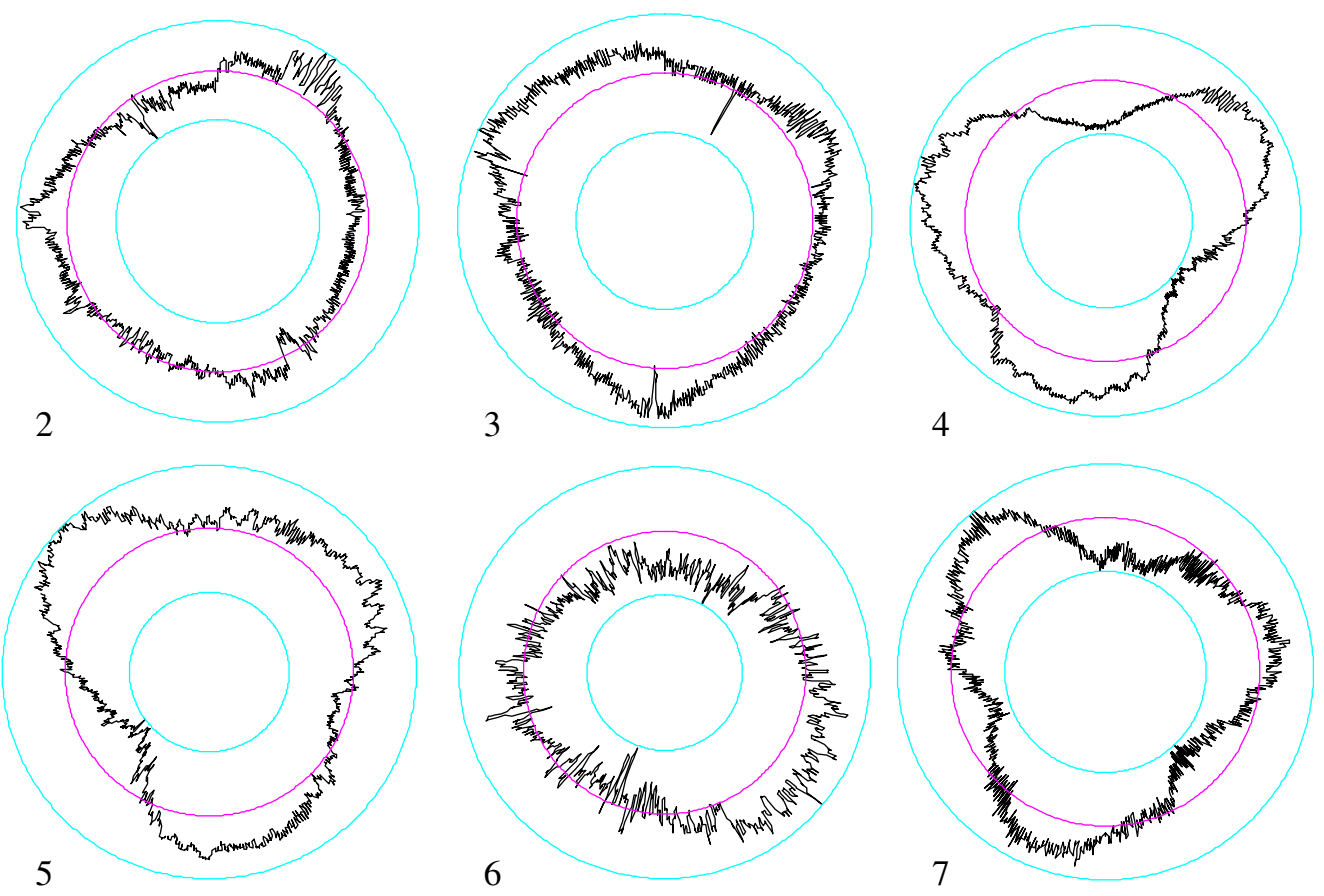

Fig. 3. Roundness deviations of samples no. 2-7 of Table 2 with the reference circles in Fig. 1. 
- E was a circle with center the LSC and radius $0.5 \mathrm{~mm}$ (about $5 \%$ of the nominal diameter).

Values of pop, $p c$ and $p m$ greater than respectively 500 , 0.9 and 0.04 have also been explored and ignored because produced no significant improvement.

\subsection{Computational experiments}

The optimization of the GA parameters has been carried out by exploring the search range defined in Table 3 .

The GA has run on the seven experimental samples of Table 2 and the average computation time on all samples has been evaluated. The graphs of Figs. 4 and 6 show the contour maps for the corresponding GA parameters indicated on the axes of the graphs. By this trial and error approach over the search space we are able to define the optimal GA parameters and point out guidelines on their

Table 3

GA parameters from the literature.

\begin{tabular}{llll}
\hline Genetic parameter & $\begin{array}{l}\text { Values } \\
\text { from [18] }\end{array}$ & $\begin{array}{l}\text { Range } \\
\text { from [25] }\end{array}$ & $\begin{array}{l}\text { Optimal values } \\
\text { from [25] }\end{array}$ \\
\hline$p o p$ & 30 & $10-50$ & 30 \\
$p c$ & 0.6 & 0.8 & 0.8 \\
$p m$ & 0.033 & $0.06,0.1$ & 0.06 \\
Generation number & 50 & $180-4000$ & 4000 \\
\hline
\end{tabular}

a For cylindricity. selection. The four graphs can also be seen as slices on the pop axis in the three dimensional search space with axes pop, $p c$ and $p m$.

Fig. 4 is the result of 784 runs of the GA by changing the parameters of $p o p, p c$ and $p m$ as designed.

It can be noticed that the computation time increases with pop in the examined range. For this reason we have shifted the optimization range below 100 .

On the other hand, lower values of the population size do not provide sufficient accuracy in the detection of the roundness error.

As an example, the case of dataset no. 1 is represented in Fig. 5. $R^{*}=0.0239 \mathrm{~mm}$ from Table 6 has been used as the target roundness error in the explorative search. It can be noticed that $R^{*}$ cannot be reached with $p o p=50$ for most $p c$ and $p m$ values as shown from the black area in Fig. 5. For pop $=70$ instead the white coverage is almost filling the examined range of $p c$ and $p m$.

Consequently pop $=70$ is assumed as the lower bound of the optimization phase discussed in the next paragraph.

\section{GA optimization}

As a consequence of the explorative phase, the GA optimization has been carried out in the range 70-100 of pop on the samples of Table 2.

The results of computational experiments for the GA optimization are shown in Fig. 6.
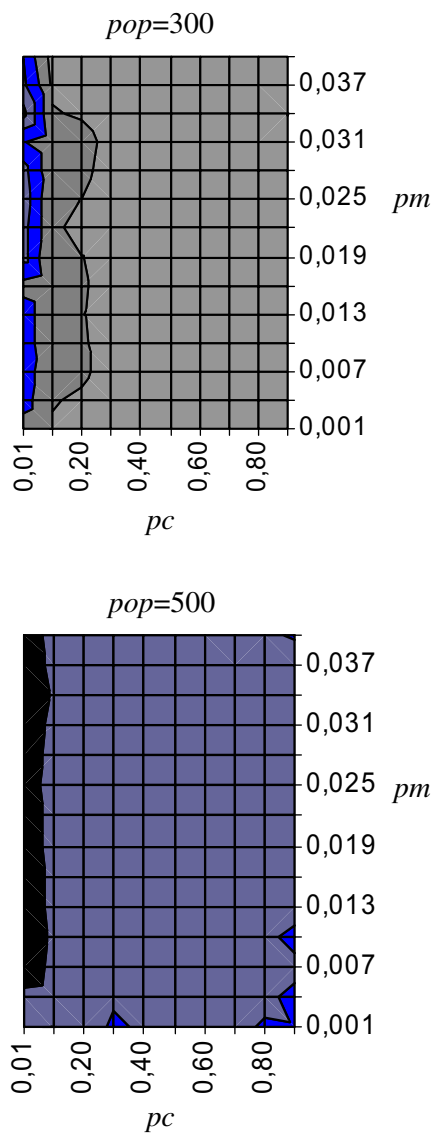

Fig. 4. GA parameters optimization: average computation time on the samples of Table 2 as a function of $p c$ and $p m$ for four different values of pop. 

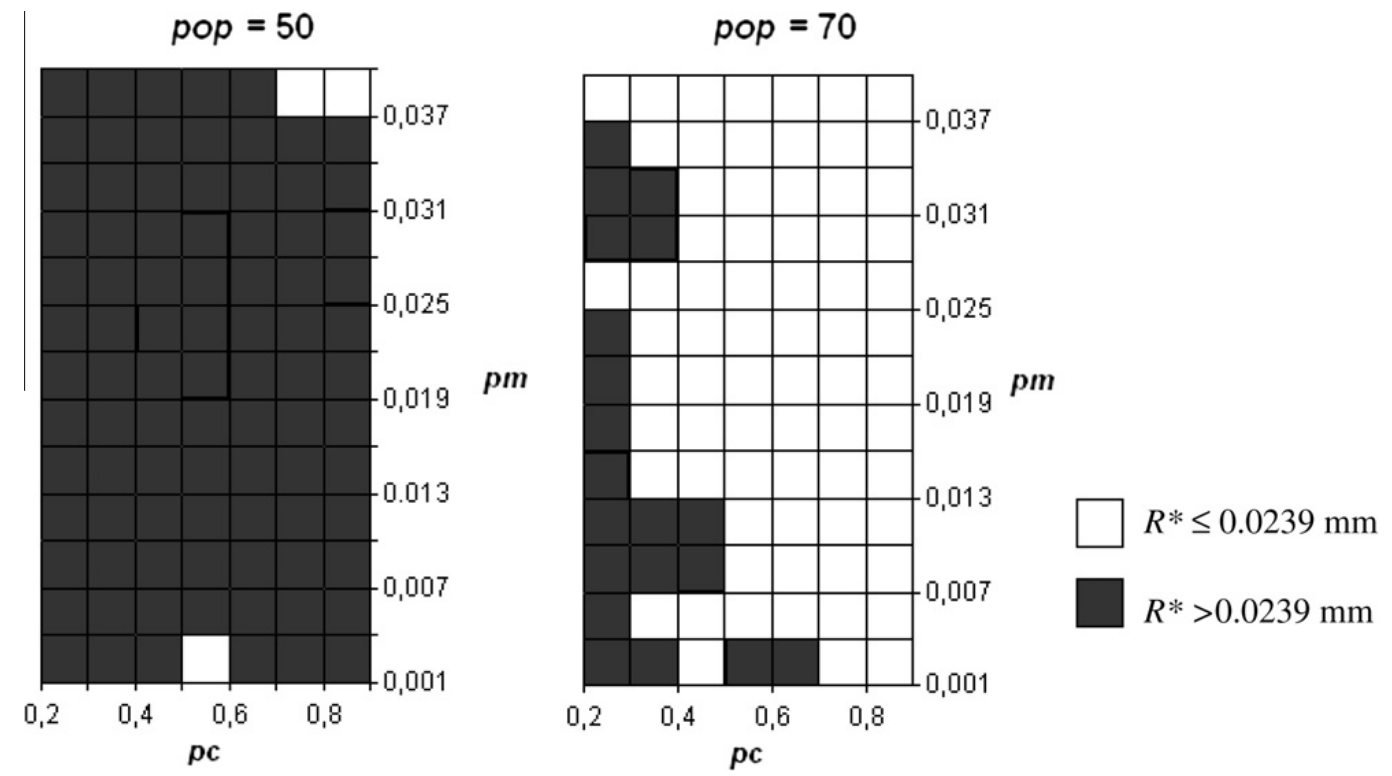

Fig. 5. GA parameters exploration: target roundness error $R^{*}$ on dataset no. 1 of Table 2 as a function of $p c$ and $p m$ for two different values of pop upon an area of promising configuration (from Fig. 4) of $p c$ and $p m$.

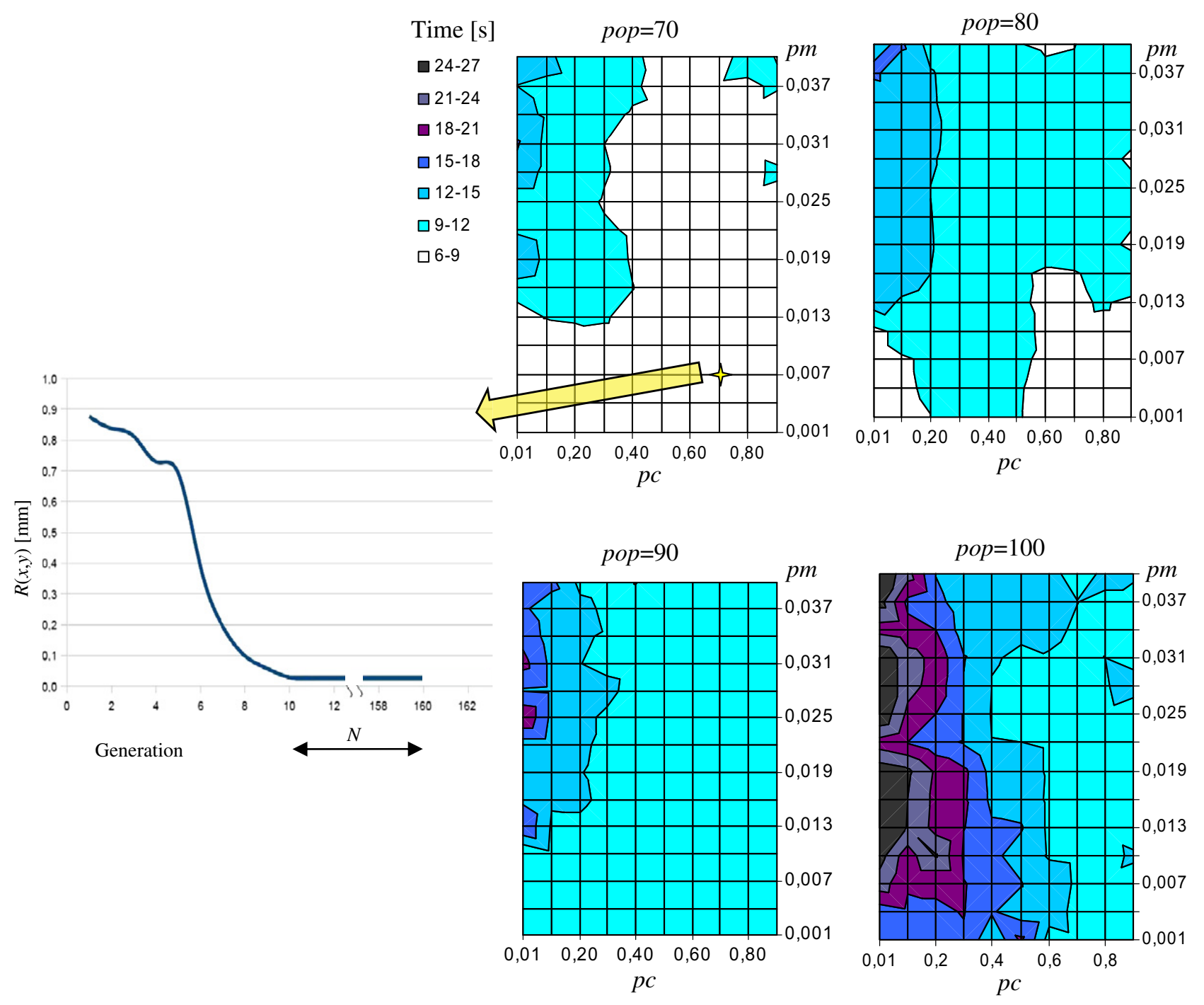

Fig. 6. GA parameters optimization: average computation time on the samples of Table 2 as a function of $p c$ and $p m$ for four different values of pop and convergence of the average roundness error for the selected parameters (left inset). 
As for Fig. 4, Fig. 6 is the result of 784 runs of the GA by changing the parameters of pop, $p c$ and $p m$ as designed. Each run has a similar shape to the one displayed in the left inset for $p o p=70, p c=0.7$ and $p m=0.07$.

\subsection{Results and discussion}

From the observation of Fig. 6 it can be noticed that varying the pop value, there is always the same optimal region for $p c$ and $p m$, and the same occurred in Fig. 4. This region is placed as specified in Table 4 .

With regard to pop, the computation time increased with this parameter, so the optimal value is the lowest one: $p o p \geqslant 70$. Smaller optimality areas are also present (brighter areas of Fig. 6).

By comparing the performance in Figs. 4 and 6 it is clear that an incorrect or random choice of the parameters can lead to an increase of the computation time by one order of magnitude, from 70 to $80 \mathrm{~s}$ to less than $9 \mathrm{~s}$.

The optimal range in Table 4 can be compared to values from the literature (Table 3) although we have worked with large samples: in particular, it can be noticed that pop increases from 30 ( 50 for cylindricity) to 70 . It has also been shown that a lower pop (e.g. 50 in Fig. 5) does not allow sufficient accuracy to the GA.

Table 4

Optimal range of GA parameters from computational experiments and selected value used in benchmark tests.

\begin{tabular}{lll}
\hline Parameter & Optimal range & Selected values \\
\hline pop & $70-80$ & 70 \\
$p c$ & $0.7-0.8$ & 0.7 \\
pm & $0.001-0.010$ & 0.007 \\
Generation number & $40-130$ & - \\
$N$ & - & 30 \\
$E$ & - & $5 \%$ of diameter \\
\hline
\end{tabular}

Table 5

Test phase. Samples obtained by certified software [26] with center coordinates $(0,0)$, number of optimal solutions found $\% \mathrm{~S}$ and computation time for 100 trials.

\begin{tabular}{|c|c|c|c|c|c|}
\hline \multicolumn{3}{|l|}{ Test data } & \multicolumn{3}{|c|}{ Computation results } \\
\hline \multirow[t]{2}{*}{ Sample size } & \multirow[t]{2}{*}{ Radius } & \multirow{2}{*}{$\begin{array}{l}\text { Maximum } \\
\text { residual } \\
\text { deviation }\end{array}$} & \multirow[t]{2}{*}{$\% \mathrm{~S}$} & \multicolumn{2}{|c|}{ CPU time (s) } \\
\hline & & & & Mean & St. dev. \\
\hline 500 & 42.000 & 0.031 & 100 & 0.46 & 0.59 \\
\hline 3600 & 20.000 & 0.020 & 100 & 4.20 & 4.63 \\
\hline 11,000 & 35.000 & 0.011 & 99 & 11.70 & 13.36 \\
\hline
\end{tabular}

\section{Table 6}

Test phase. Roundness errors on the seven samples of Table 2 (single run with optimal GA parameters obtained from 1568 runs). Computation time for the LSQ method is approximately zero for all samples.

\begin{tabular}{llll}
\hline Samples & LSE $(\mathrm{mm})$ & MZE $(\mathrm{mm})$ & CPU time $(\mathrm{s})$ \\
\hline 1 & 0.0242 & 0.0239 & 2.6 \\
2 & 0.0299 & 0.0262 & 2.6 \\
3 & 0.0292 & 0.0273 & 1.3 \\
4 & 0.0668 & 0.0666 & 1.0 \\
5 & 0.0725 & 0.0667 & 7.4 \\
6 & 0.0934 & 0.0925 & 2.7 \\
7 & 0.0423 & 0.0391 & 1.1 \\
\hline
\end{tabular}

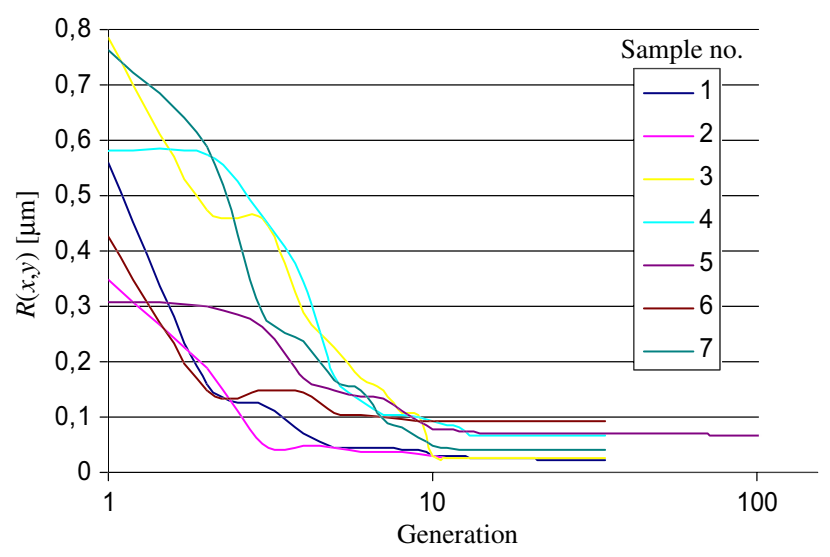

Fig. 7. Convergence of MZT for the seven samples of Table 2 with the selected values of the optimal parameters in Table 4.

The pop increase from 30 to 70 seems a consequence of the larger dataset.

The mutation probability pm also shows a significant change, three times lower and over.

The reduction of $p c$ or $p m$ can be considered as a beneficial effect, because it reduces the effect of the parameter itself and the "entropy" of the algorithm. A lower $p c$ or $p m$ reduces the random search and enforces the solution search in fewer areas with higher success probability. Lower crossover and mutation is then beneficial for the convergence speed with user defined accuracy because fewer search areas are involved. It is then speculated that the dataset size increase is beneficial for the GA stability. If a larger dataset implies a lower pm (like in the current case) and a lower pm provides higher stability to the algorithm, it could be concluded that with larger samples the search is less random and driven by the most promising areas. This looks reasonable because of the higher amount of information involved.

Fig. 7 shows the convergence of the algorithm on the seven samples with the selected values of the optimal parameters in Table 4.

The behavior of the convergence curve changes for the different samples, because of their different nature. However, already after 10 generations, their average represents the general trend within a range of $0.8 \mu \mathrm{m}$. From Fig. 7 it can also be noticed that the major improvements on $R(x$, $y$ ) are concentrated in the first few tenths of generations, particularly before 100 .

As far as the stop criterion is concerned, it has been observed that the speed of convergence of the roundness error is lower than $0.1 \mu \mathrm{m}$ per 16 generations. By increasing $N$ over 16 the computation time increases without significant accuracy improvement nevertheless $N$ can be conservatively set to 30 as reported in Table 4 .

For applications where the accuracy matters more than the computation time (e.g. prototypical versus mass production) $N$ can be increased accordingly.

\section{Benchmark tests}

Three samples [24] obtained by certified software [26] have been used to validate the proposed GA with the high 
Table 7

Comparison with other samples and optimization algorithms from the literature.

\begin{tabular}{llll}
\hline Data & Methods & MZE (mm) & CPU type \\
\hline Benchmark no. 1 & Ref. [2] & 0.0085 & Exact method \\
139 sample points & Proposed GA & 0.0085 & Intel Core 2 Duo \\
& GA [19] & 0.00 & CMM processing unit \\
& EGA [19] & 0.05 & 0.28 \\
Renchmark no. 2 & Ref. [27] & 0.0106 & CMM processing unit \\
100 sample points & Proposed GA & 0.9574 & Intel Pentium III \\
& GA [19] & 0.9649 & Intel Core 2 Duo \\
& EGA [19] & 0.9574 & CMM processing unit \\
Renchmark no. 3 & Ref. [8] & 1.0866 & CMM processing unit \\
24 sample points & Proposed GA & 0.0382 & Celeron 366 \\
& GA [19] & 0.0382 & Intel Core 2 Duo \\
Benchmark no. 4 & EGA [19] & 0.0382 & CMM processing unit \\
25 sample points & Ref. [8] & 0.0405 & 0.00 \\
& Proposed GA & 0.03 & 0.025 \\
\end{tabular}

success rate shown in Table 5 . It seems that the GA accuracy is not influenced by increasing the number of sample points. With thousands of sample points, with current processors, the computation time with the proposed GA is in the order of the second, which is acceptable by current industrial practice.

The GA results algorithm with the selected values of the optimal parameters in Table 4 has been reported in Table 6 with the reference LSE obtained with the LSQ method.

The computation time in Table 6 is related to the stability condition of $N=30$ hence the total amount of processed generations are between 40 and 130 (Table 4).

From Table 6 it can be noticed that the better roundness error estimation is achieved by paying a low computation time.

The proposed GA has been compared with four samples from the literature as detailed in Table 7. Unfortunately no large samples are available (thousands sample points versus less than hundred).

Table 7 summarizes the optimal results achieved by other researchers. For each benchmark, the first values have been determined by the author of the original dataset with its proposed method $[2,8,27]$. The other three methods for benchmarks no. 1-3 are GAs, for comparison within the same class of methods. Benchmark no. 4 has been added to increase the test set.

In benchmarks no. 1 and no. 3 the MZE of the proposed GA matches that of the better GA [19] and that of the author of the original samples $[2,8]$.

The MZE with the proposed GA on benchmark no. 2 is $0.0075 \mathrm{~mm}$ higher than that of the better GA [19] and that of the author of the original dataset [27].

In benchmarks no. 1-3 the MZE of the proposed GA is lower than that of the EGA in [19].

The MZE with the proposed GA on benchmark no. 4 is $0.0002 \mathrm{~mm}$ higher than that of the author of the original dataset [8].

The proposed GA shows lower roundness error with benchmarks no. 1, 3 and 4. Consequently, the worst performance of the proposed GA on the benchmark no. 2 seems related to its higher roundness error (almost $1 \mathrm{~mm}$ ). With this assumption, the proposed GA is more suitable for the inspection of more accurate manufacturing operations.
The CPU times in Table 7 are not directly comparable because of the different processor type. However, considering that they are in the order of $1 \mathrm{~s}$, all listed algorithms seem suitable for actual manufacturing needs.

Considering the optimal result on the small dataset from [19], where the GA ran without mutation, and that the computational experiments in all the search range ( $p o p=70-500$ ) detected the same optimal region for $p c$ and $p m$ where $p m$ was in the lower bound, it can be concluded that mutation is not a fundamental operator for the examined problem.

\section{Conclusion}

In this paper a fast algorithm for accurate evaluation of roundness errors with large samples according the MZT criterion has been developed.

The GA parameters optimization has been carried out on seven samples starting from initial GA parameters taken from the literature.

Computational experiments have pointed out the following outlook:

- larger dataset require higher population size values as preliminarily conjectured;

- the literature values for the probability of crossover have not been significantly affected by the larger dataset;

- the mutation probability decreases with larger samples because of the higher amount of information on the involved part. It is also conjectured that mutation is not a fundamental operator for the examined problem;

- a stability condition has been imposed corresponding to a convergence speed greater than $0.1 \mu \mathrm{m}$ per 30 generations and the stop criterion determined.

Simulations have shown that the optimal genetic parameters lead to a significant decrease (one order of magnitude) of the computation time (from 70 to $80 \mathrm{~s}$ to less than $9 \mathrm{~s}$ ).

The proposed GA with the optimal parameters has also been validated with larger samples obtained by certified 
software and has been compared with benchmarks from the literature, necessarily smaller, showing its effectiveness.

The proposed GA can be directly applied in the shop floor to achieve the economic benefits claimed by using the scheme (Fig. 2) and parameters (Table 4) described in the paper.

By reducing the computation time of the minimum zone tolerance method, the most accurate method to evaluate roundness errors can be profitably applied real-time (on-line).

The higher speed of convergence on large samples allows the application of the MZT to CMM scanning techniques, which involve thousands of sample points. The optimum dataset size for a given target accuracy requires further investigation.

Future work includes the investigation of different genetic mechanisms like roulette wheel selection or knowledge-augmented crossover, neglecting mutation.

To assess the generality of the optimal GA parameters found and selection guidelines, they will be tested with other form tolerances, like cylindricity, straightness and flatness and with incomplete geometric features.

\section{Acknowledgments}

This work has been developed as teaching project of the students Antonetti and Barloscio for the class of manufacturing processes II of the master degree in mechanical engineering at the School of Engineering of the University of Pisa.

\section{References}

[1] A.B. Forbes, Least-squares best-fit geometric elements, NPL Rep. DITC 140/89, National Physics Laboratory, UK, April 1989.

[2] W.Y. Jywe, C.H. Liu, C.K. Chen, The min-max problem for evaluating the form error of a circle, Measurement 26 (1999) 273-282.

[3] G. Goch, K. Lubke, Tschebyscheff approximation for the calculation of maximum inscribed/minimum circumscribed geometry elements and form deviations, CIRP Annals - Manufacturing Technology 57 (1) (2008) 517-520.

[4] International Organization for Standardization, Geneva, Switzerland, ISO/TS 12181-1: Geometrical Product Specifications (GPS) Roundness - Part 1: Vocabulary and Parameters of Roundness, 2003.

[5] International Organization for Standardization, Geneva, Switzerland, ISO 1101, Geometrical Product Specifications (GPS) - tolerances of form, orientation, location and run out, second ed., December 2004.

[6] A. Rossi, A form of deviation-based method for coordinate measuring machine sampling optimization in an assessment of roundness,
Proceedings of Institution of Mechanical Engineers, Part B: Journal of Engineering Manufacture 215 (2001) 1505-1518.

[7] A. Rossi, A minimal inspection sampling technique for roundness evaluation, in: First CIRP International Seminar on Progress in Innovative Manufacturing Engineering (Prime), Sestri Levante, Italy, June 2001, pp. 411-416.

[8] L.M. Zhu, H. Ding, Y.L. Xiong, A steepest descent algorithm for circularity evaluation, Computer Aided Design 35 (2003) 255-265.

[9] T.S.R. Murthy, S.Z. Abdin, Minimum zone evaluation of surface, International Journal of Machine Tool Design and Research 20 (2) (1980) 123-136.

[10] Y.L. Xiong, Computer aided measurement of profile error of complex surfaces and curves: theory and algorithm, International Journal Machine Tools and Manufacture 30 (1990) 339-357.

[11] J. Lai, I. Chen, Minimum zone evaluation of circles and cylinders, International Journal of Machine Tools and Manufacturing 36 (4) (1995) 435-451.

[12] M. Wang, S.H. Cheraghi, A.S.M. Masud, Circularity error evaluation: theory and algorithm, Precision Engineering 23 (3) (1999) 164-176.

[13] Y. Wang, Application of optimization techniques to minimum zone evaluation of form tolerances, quality assurance through integration of manufacturing process, systems, ASME PRD (1992) 56.

[14] G.L. Samuel, M.S. Shunmugam, Evaluation of circularity from coordinate and form data using computational geometric techniques, Precision Engineering 24 (2000) 251-263.

[15] H. Chang, T.W. Lin, Evaluation of circularity tolerance using Monte Carlo simulation for coordinate measuring machines, lnternational Journal of Production Research 31 (1992) 2079-2086.

[16] U. Roy, X. Zhang, Establishment of a pair of concentric circles with the minimal radial separation for assessing roundness error Computer Aided Design 24 (2) (1992) 161-168.

[17] L. Xianqing, Z. Chunyang, X. Yujun, L. Jishun, Roundness error evaluation algorithm based on polar coordinate transform, Measurement 44 (2) (2011) 345-350.

[18] R. Sharma, K. Rajagopal, S. Anand, A genetic algorithm based approach for robust evaluation of form tolerances, Journal of Manufacturing Systems 19 (1) (2000) 46-57.

[19] X. Wen, Q. Xia, Y. Zhao, An effective genetic algorithm for circularity error unified evaluation, International Journal of Machine Tools and Manufacture 46 (2006) 1770-1777.

[20] J. Mao, Y. Cao, J. Yang, Implementation uncertainty evaluation of cylindricity errors based on geometrical product specification (GPS), Measurement 42 (5) (2009) 742-747.

[21] C.M. Shakarji, A. Clement, Reference algorithms for Chebyshev and one-sided data fitting for coordinate metrology, CIRP Annals Manufacturing Technology 53 (1) (2004) 439-442.

[22] J. Holland, Adaptation in Natural and Artificial System, The University of Michigan Press, Ann Arbor, MI, 1975.

[23] K.A. DeJong, Analysis of the behavior of a class of genetic adaptive systems, PhD thesis, University of Michigan, USA, 1975.

[24] http://www.dimnp.unipi.it/lanzetta/mzt.

[25] H.-Y. Lai, W.-Y. Jywe, C.-K. Chen, C.-H. Liu, Precision modeling of form errors for cylindricity evaluation using genetic algorithms, Precision Engineering 24 (2000) 310-319.

[26] National Physical Laboratory (UK), Data Generator for Chebyshev Best-Fit Circle. <http://www.npl.co.uk/mathematics-scientificcomputing/software-support-for-metrology/> (accessed November 2011).

[27] J. Huang, A new strategy for circularity problems, Precision Engineering 25 (2001) 301-308. 